\title{
How to handle the inelastic collapse of a dissipative hard-sphere gas with the TC model
}

\author{
Stefan Luding $^{(1)}$ and Sean McNamara ${ }^{(1,2)}$
}

\begin{abstract}
The inelastic hard sphere model of granular material is simple, easily accessible to theory and simulation, and captures much of the physics of granular media. It has three drawbacks, all related to the approximation that collisions are instantaneous: 1 ) The number of collisions per unit time can diverge, i.e. the "inelastic collapse" can occur. 2) All interactions are binary, multiparticle contacts cannot occur and 3) no static limit exists. We extend the inelastic hard sphere model by defining a duration of contact $t_{c}$ such that dissipation is allowed only if the time 'between contacts is larger than $t_{c}$. We name this generalized model the TC model and discuss it using examples of dynamic and static systems. The contact duration used here does not change the instantaneous nature of the hard 'sphere contacts, but accounts for a reduced dissipation during "multiparticle contacts". Kinetic and elastic energies are defined as well as forces and stresses in the system. Finally, we present event-driven numerical simulations of situations far beyond the inelastic collapse, possible only ' with the TC model.
\end{abstract}

\section{Contents

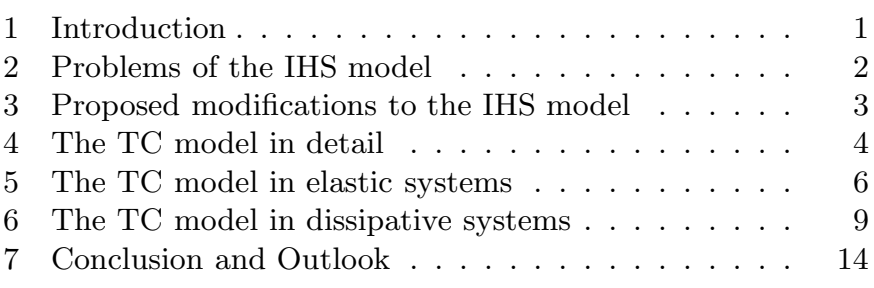

Received: June 29, 1998

Stefan Luding ${ }^{(1)}$ and Sean McNamara ${ }^{(1,2)}$

(1) Institute for Computer Applications 1,

Pfaffenwaldring 27,

70569 Stuttgart, GERMANY

e-mail: lui@ica1.uni-stuttgart.de

(2) Levich Institute,

Steinman Hall T-1M,

140th St and Convent Ave,

New York, NY 10031, USA

e-mail: mcnamara@levdec.engr.ccny.cuny.edu

We thank Timo Aspelmeier, Jean Rajchenbach, Stefan Schwarzer, and Annette Zippelius for helpful discussions and acknowledge the support of the "Alexander von Humboldt-Stiftung" and of the Deutsche Forschungsgemeinschaft, Sonderforschungsbereich 382 .
1

\section{Introduction}

Granular media consist of discrete particles. Their interaction is governed by two major concepts: excluded volume and dissipation. Since the particles are solid, each particle occupies a certain amount of space, and no other particle may enter this volume. If another particle approaches, the pair eventually collides. During collisions, energy is lost from those degrees of freedom (linear or rotational motion) which are important for the behavior of the material. Heat or sound are radiated and plastic deformation takes place so that energy is irreversibly lost [1].

A model accounting for both excluded volume and dissipation is the inelastic hard-sphere (IHS) molecular dynamics with dissipative binary interactions. It is frequently used for the simulation of granular media, and is attractively simple. Between collisions, particles move freely through space. When two particles touch, their velocities are instantly replaced by new velocities calculated from a collision rule:

$\mathcal{U}^{\prime}=\mathcal{C}(\mathcal{U}, \mathcal{R})$,

where $\mathcal{U}$ are the particles' velocities before the collision, and $\mathcal{U}^{\prime}$ are those after the collision. $\mathcal{R}$ denotes the particles' positions at the time of collision. In theory, $\mathcal{C}$ could be anything, but in practice it is restricted by physical considerations, i.e. the particles may not interpenetrate, Galilean invariance, conservation of momentum, dissipation of energy, etc.. $\mathcal{U}$ may also contain angular velocities, and $\mathcal{C}$ can be chosen to mimic real particles. Simulations using the hard sphere model can be very fast because to simulate a collision, the computer needs only to evaluate Eq. (1). On the other hand, if forces between particles are specified, the computer must integrate a differential equation over several time steps for each collision. Note that the assumption of an ideally hard potential is also used in kinetic theory and the Boltzmann or Enskog approaches [2 [- , which facilitates comparisons between simulation and theory. All the collision rules we consider in this paper can be written

$\mathbf{v}_{1,2}^{\prime}=\mathbf{v}_{1,2} \pm \frac{1+r}{2}\left[\left(\mathbf{v}_{2}-\mathbf{v}_{1}\right) \cdot \hat{\mathbf{n}}\right] \hat{\mathbf{n}}$

where $\hat{\mathbf{n}}$ is a unit vector joining the line of centers, and $\mathbf{v}_{i}$ is the velocity of particle $i$. But the most important symbol appearing in Eq. (2) is $r$, the restitution coefficient. The following equation for $r$ can be derived from Eq. (2):

$r=-\frac{\left(\mathbf{v}_{2}^{\prime}-\mathbf{v}_{1}^{\prime}\right) \cdot \hat{\mathbf{n}}}{\left(\mathbf{v}_{2}-\mathbf{v}_{1}\right) \cdot \hat{\mathbf{n}}}$ 
so $r$ is the ratio of the component of the relative velocity along the line of centers after the collision to its value before the collision. If $r=1$, collisions conserve energy, and are said to be elastic. For $0 \leq r<1$, energy is dissipated, and the collisions are inelastic. Usually $r$ is considered to be a property of the material, and set to a constant which is the same for all collisions.

The IHS model is best accessible to simulations and theory, but it has three general problems. First, there is the singularity of inelastic collapse: an infinite number of collisions can occur in finite time. Secondly, the collision rule treats only binary interactions, but in reality, many grains can interact, and these multiparticle interactions are different from a sequence of binary collisions. Finally, there are no enduring contacts between particles, and no analog to various physical quantities, such as the energy stored in inter-particle contacts, exists. In this paper, we present the "TC model", which is an extension of the IHS model that remedies these three problems. The collisions are still instantaneous, but we suppose that two particles influence each other during a time $t_{c}$ after the collision. Specifically, if a particle experiences two collisions separated by a time less than $t_{c}$, a multiparticle event is assumed to occur, and the second collision dissipates no energy. Except for this additional rule, the TC model is identical to the IHS model.

The problems of the IHS model are discussed in more detail in Sec. II. In Sec. III, we review various attempts to solve these problems, including the TC model. Sec. IV describes the TC model in detail and applies it to the simple example of a particle lying on a flat surface. The elastic energy of a two-dimensional hard sphere gas is defined in section V and in section VI results on dissipative systems are presented that could be achieved by using the TC model whose consequences and future perspectives are discussed in section VII.

\section{2}

\section{Problems of the IHS model}

In this chapter, we discuss the problems of the IHS model that any improvement of it would have to correct. All three problems have essentially one origin: the potential used between the centers of mass of two colliding particles is unphysically stiff. The instantaneous collisions imply an interaction potential which is constant when there is no contact, and suddenly becomes infinite when the particles touch. Therefore, momentum exchange takes place in zero time and thus the corresponding forces are infinite, however, acting for zero time only. In a real system the situation is different: each contact takes a finite time during which large, but finite forces act. The infinitely stiff hardsphere interaction is only an idealization or simplification of a smooth repulsive pair-potential.

\section{1}

\section{Inelastic Collapse}

The most dramatic consequence of the infinitely stiff interaction potential used in the IHS model is inelastic collapse, which manifests itself as an infinite number of collisions in finite time. It was first discovered while studying the one-dimensional (1D) model system of a column of dissipative particles hitting a wall. The occurence of the inelastic collapse can be estimated using the product of the number of particles $N$ and the dissipation per contact $(1-r)$. The effective dissipation $\xi=N(1-r)$ has a critical value of $\xi_{c} \approx \pi$ above which collapse occurs. The above value of $\xi_{c}$ was calculated with the independent collision wave (ICW) model [7]. With slightly different arguments using the "cushion model" [8], the value was evaluated as $\xi_{c} \approx \ln [4 /(1-r)]$. The ICW model seems to work better in the inelastic limit, whereas the cushion model is superior in the elastic limit $[8]$.

Inelastic collapse is also present in two dimensions (2D). In freely cooling systems a minimum of three particles is enough to lead to the collapse, if dissipation and density are large enough 19, 10]. In larger assemblies, the inelastic collapse occurs, but it involves just a few particles arranged almost along a line. This leads to the conclusion that the inelastic collapse is mainly a 1D effect [1] and that the one-dimensional predictions for the critical $\xi$ should work also in $2 \mathrm{D}$. In fact, inelastic collapse in 2D unforced simulations can be predicted reasonably well by using the $1 \mathrm{D}$ criteria with $\xi=(\nu l / d)(1-r)$ ( $\nu$ is the fraction of the total area covered by the disks, $l$ is the lenght of one side of the domain, and $d$ is the disk diameter) [12]. In a container in the presence of gravity, this expression for $\xi$ is equivalent to the number of layers of particles when the granular material is at rest. With these boundary conditions, the inelastic collapse likely occurs for small energy input and large $\xi[13]$. Vibrated containers with large filling heights cannot be simulated with the IHS model 13, 14.

In two dimensions only a small fraction of the particles in the system is involved in the inelastic collapse. This implies that it is both physically insignificant for real particle assemblies and a major drawback for numerical simulations of dissipative systems. Therefore, any improvement of the IHS model must avoid the singularity of inelastic collapse.

\section{2 Multiparticle interactions}

In the IHS model, true multiparticle interactions are impossible because collisions are instantaneous. Multiparticle interaction is built up out of many two-particle interactions. In a real system the situation is different: Each contact takes a finite time so that multiparticle contacts are possible. The difference between two- and multiparticle contacts was examined for one- and two-dimensional model systems 15 17, and it was found that less energy is dissipated in multiparticle events than in an equivalent sequence of binary collisions. Any improvement of the IHS model should have the property that energy dissipation is reduced during multiparticle interactions.

\section{3}

\section{No static limit}

Another problem with the IHS model is that the static limit does not exist, i.e. there is no way to represent endur- 
ing contacts between particles. For example, in the framework of the IHS model, a particle cannot rest motionless on the ground. We discuss this simple example in more detail in subsection 4.2. Another way to formulate this problem is by considering the energies in the system, see also subsection 4.1, and especially the elastic contact energy that is not defined in the IHS model.

The translational kinetic energy $E$ of the system is $E=(1 / 2) \sum_{i=1}^{N} m_{i}\left(v_{i}^{2}+u_{i}^{2}\right)$, with the mass $m_{i}$, the fluctuation velocity $v_{i}$, and the flux velocity $u_{i}$ of particle $i$. In the following we will mainly consider situations with $u_{i}=0$. The potential energy $E_{p}$ is zero in absence of an external body force like e.g. gravity. In addition to $E$ and $E_{p}$, real materials have an elastic energy $E_{e l}$ at each contact, which is not defined in the context of the IHS model. In classical elastic systems, the total energy, i.e. the sum of kinetic, potential, and elastic energy, $E+E_{p}+E_{e l}=E_{\text {tot }}$, is always conserved. In a dissipative system without a source of energy, the total energy tends towards a constant while the kinetic energy tends towards zero in the long time limit. This state is referred to as static, not to be confused with a "quasi-static" state, defined in the context of the TC model below in subsection 4.1.

More specifically, consider a realistic granular material inside a box, under the influence of gravity and in the absence of energy sources. Due to dissipation, the material will loose energy. The potential and elastic energies will approach constants values while the kinetic energy tends towards zero. Eventually all particles are at rest, with $E=0$, and the elastic energy is, as a rule, larger than zero, since a certain number of contacts is necessary to allow for a stable static configuration. If the particles would be dissipative hard spheres, the inelastic collapse can occur long before $E$ vanishes so that the system will never reach a configuration with constant $E_{\text {tot }}$. The inelastic collapse brings the system to an artificial halt. A static configuration with zero kinetic energy could be reached in a hard-sphere system by piling the spheres on top of each other (just in contact with no overlap). For this situation $E_{e l}$ is not well defined and those touching hard spheres violate the rule of instantaneous contacts. Since in this artificial limit no elastic energy is defined, contactforces and stresses are also not properly defined. This is an argument against the IHS model itself, which has by construction no static or "zero-temperature" limit.

A method that loosens the restriction of instantaneous contacts and in return defines contact forces is the socalled contact dynamics, (see Ref. 18 and references therein). The TC model, which we present in this paper, also provides a way to define the elastic energy.

\section{3}

\section{Proposed modifications to the IHS model}

In this section we review the various extensions and modifications of the IHS model which have been proposed. The main goal of these suggestions has been to remove inelastic collapse, since it is the most conspicuous problem of the IHS model.

(i). Particles with relative energy below a critical threshold can be merged into a "cluster" by setting their rela- tive velocity and separation to zero. If another particle hits such a cluster, the momentum transport inside the cluster takes place instantaneously in the sequence of the largest relative velocity (LRV) [19]. With this method clusters can grow, and, given strong enough energy input, clusters may also be destroyed again. The deterministic LRV model has been successfully implemented in $1 \mathrm{D}$, but stresses and energies in the bulk are not defined.

(ii). Several authors suggest a stochastic addition of translational or rotational energy, as soon as the relative velocity after a collision drops below a critical value [13,20]. Also a small rotation of the relative velocity after contact of less than 5 degrees, seems to hinder the inelastic collapse [20], since correlations between successive collisions are diminished. Those stochastic models prevent the occurence of the inelastic collapse in the systems examined. However, neither is it clear whether the collapse can be circumvented under all conditions, nor has the physical relevance of the random energy input been discussed so far.

(iii). Another method involves internal modes of every particle. At each collision these modes may be agitated, and their energy is dissipated only on a time-scale longer than the duration of a contact. If a particle suffers an additional collision within this time, then energy can be transferred from the internal modes back into translational motion. At least in a cooling system in 1D the inelastic collapse is prevented [21,22], and possibly this model leads to an explanation of the random energy input mentioned above (ii). The drawback of this method is the effort necessary to model the internal modes.

(iv). A frequently used way to reduce dissipation in the low velocity regime (which has also been observed experimentally) is a velocity dependent restitution coefficient $r(v)$ based on the assumption of either viscoelastic [15, 23] or plastic [24, 25] contacts. The velocity dependence seems to avoid the inelastic collapse in the absence of walls and external forces [10], however, its use for other boundary conditions resembling systems on earth, has not been examined up to now. Note that the dependence of $r(v)$ on the collision velocity still concerns binary collisions with varying velocity, whereas the TC model discussed below concerns the transition from binary to multiparticle contacts - a completely different approach. Even when a velocity dependence of $r$ can simply be added to the TC model, we avoid this in the following for the sake of simplicity.

(v). Instead of feeding energy into the system, another approach just switches off dissipation following certain rules. The idea is to decide whether a particle still feels its previous collision partner when colliding with the next one. This is important since the presence of a third particle will affect the collision of a given pair. One can switch off dissipation if the next collision partner of a particle is detected within a critical distance 
$\lambda_{c}$ [12]. In fact, it makes no sense to treat particles as separate objects if their surface-surface distance is of atomic size and, more technically, the numeric errors can become large when the distance between the particles is many orders of magnitude smaller than the particle diameter.

A similar argument for switching off dissipation is that two collisions cannot be treated as separate events if they take place within a too short time-interval $t_{c}$ that corresponds to the duration of a contact. It has been shown that collisions which overlap (in time) lead to weaker dissipation than a series of binary collisions (separated in time) [15, 16, 26]. Thus one sets the restitution coefficient to its elastic limit $r=1$, if a particle suffers more than one collision within time $t_{c}$ [14, 27, 28]. With the time $t_{n}^{(i)}$, that passed by since the last collision $n-1$ of particle $i$, the restitution coefficient for its $n$-th collision can be expressed as

$r_{n}^{(i)}= \begin{cases}r & \text { for } t_{n}^{(i)}>t_{c} \\ 1 & \text { for } t_{n}^{(i)} \leq t_{c},\end{cases}$

with $0<r \leq 1$. Thus the type of a collision changes from inelastic to elastic when collisions occur too frequently. More specifically, a collision is elastic if at least one partner fulfills the above condition $t_{n}^{(i)} \leq t_{c}$. In general, $r$ and $t_{c}$ can depend on the relative velocity and other parameters [15, 23], so that the material's behavior can be adjusted appropriately using this dependence as discussed above (iv). Since Eq. (11) involves the duration of a contact $t_{c}$ we refer to this model as TC model in the following.

Before turning to some more details of the TC model, we should mention that by using a velocity dependence for either the dissipation cut-off distance $\lambda_{c}$ or the dissipation cut-off time $t_{c}$, both can be connected: Assuming $t_{c}(v) \propto v^{-\alpha}$, with the typical relative velocity $v$, leads to $\lambda_{c} \propto v t_{c}(v) \propto v^{1-\alpha}$. With $\alpha=1$ one has $\lambda_{c}=$ const, and alternatively, with $\lambda_{c} \propto v$ one gets $t_{c} \propto \lambda_{c} / v=$ const.. This unifies both the critical time and the critical length criteria into a single framework. In the following sections we will focus on the cut-off time (or contact duration) $t_{c}$.

To our knowledge, none of the models mentioned above has a solid theoretical background, except for the approach in (iii) involving internal modes. The LRV method (i) has no reasonable static limit where stresses can be defined. The stochastic approaches (ii) require the choice of an a priori unknown source of fluctuation energy. The velocity dependence of $r$ in (iv) was experimentally measured for binary collisions, and is not necessarily important for multiparticle contacts. In the following we will discuss the physical relevance of the TC model, define its quasi-static limit, and finally apply it to selected examples.

\section{4}

\section{The TC model in detail}

In Fig. 1, an interaction of two 'soft' particles (left) is compared to the interaction of two 'hard' particles (right).
The words 'hard' and 'soft' correspond to non-smooth and smooth potentials between the centers of mass of two particles respectively, and both are different approaches to model solid body interaction. Soft particles interact essentially during a time $t_{c}$, indicated by the shaded region and the dashed vertical lines which mark the beginning and the ending of the contact. The contact duration $t_{c}$ of the soft particles is defined by these two instants of time. In the case of the hard particles, the interaction is instantaneous and the beginning and the ending coincide. However, in the TC model, the particles are considered to influence each other also during a time $t_{c}$ after each collision (the shaded region). Note that the TC model in the limit $t_{c}=0$ is identical to the IHS model and that both are identical to the elastic hard sphere model when $r=1$.
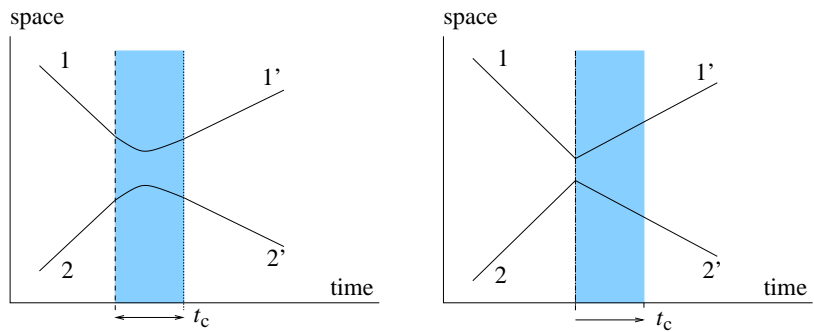

Fig. 1. Schematic plot of the trajectories of two soft (left) and two hard (right) particles against time. The beginning and the ending of the interaction are marked by dashed and dotted vertical lines respectively and the time $t_{c}$ during which dissipation is affected is marked as shaded region.

Assuming that $t_{c}$ is the physical duration of a contact, multiparticle contacts take place whenever a further collision of the particles in Fig. Al(b) occurs within the shaded area. This is the case when the typical time between instantaneous contacts $t_{n}$ gets smaller than the duration of a contact $t_{c}$. The ratio $\tau_{c}=t_{n} / t_{c}$ is a measure for the existence of multiparticle contacts $[15]$. If $\tau_{c} \gg 1$ pair interactions dominate, whereas for $\tau_{c} \ll 1$, one particle may be in contact with several others. Numerical simulations with various soft interaction potentials show that dissipation gets more and more ineffective with decreasing $\tau_{c}$ [15, 16$]$.

\section{1}

\section{The energies in the system}

In this subsection, we show how an elastic energy can be defined in the TC model. As was discussed above, there is no elastic energy in the IHS model. In a real system, only particles in contact with each other contribute to the elastic energy. As a consequence, we consider all particles which collided no longer than $t_{c}$ ago to be still in contact, and thus their energy contributes to a pseudo-elastic energy $E_{e}$. The translational kinetic energy $E$ splits into a free kinetic energy $E_{k}$ and the pseudo-elastic energy $E_{e}$ so that $E_{k}=E-E_{e}$. Free means that the kinetic energy $E_{k}$ can be dissipated whereas elastic energy $E_{e}$ cannot. In 
both the IHS and TC models, $E_{e l}$ does not exist due to the non-smooth interaction potential, but in the TC model, it is replaced by $E_{e}$. In table 11 we summarize the meaning of the symbols in the framework of the different models. From table 1 it is obvious that some type of elastic energy is missing in the IHS model, while the TC model has three types of energy like a real system. However, a direct quantitative comparison of the energies in the framework of a realistic system to those in the TC model is far from the scope of this study 29].

\begin{tabular}{lccc}
\hline & Real & IHS & TC \\
\hline \hline (free) kinetic energy & $E$ & $E$ & $E_{k}$ \\
\hline contact energy & $E_{e l}$ & 0 & 0 \\
elastic, kinetic energy & 0 & 0 & $E_{e}$ \\
\hline potential energy & $E_{p}$ & $E_{p}$ & $E_{p}$ \\
\hline
\end{tabular}

Table 1. Meaning of the symbols $E, E_{k}, E_{e l}, E_{e}$, and $E_{p}$ in the framework of a real system (or soft particle model), the classical hard sphere model (IHS) or the TC model.

As already mentioned in subsection 2.3 the static limit of a real system is reached when $E=0$, and $E_{e l}+E_{p}=$ const. . The corresponding state of the TC model is, consequently, identified by $E_{k}=0$, and $E_{e}+E_{p}=$ const.. However, we denote it as the "quasi-static" limit, because $E_{e}>0$ implies translational motion due to the kinetic nature of the elastic energy $E_{e}$. A similar situation with $E=0$, and $E_{p}=$ const. in the framework of the IHS model would mean that the system is artificially frozen.

For example, consider a periodic system with realistic dissipative particles and without external forces $\left(E_{p}=0\right)$ in its center of mass reference frame. Starting from an initial configuration with $E>0$ the system will evolve in time and the energy will decay. Note that this boundary conditon is totally different from the situation discussed in section 2.3 when gravity was active and walls were present. The only stable static situation (in the sense that $E_{\text {tot }}=$ const.) would be one with all particles at rest $(E=0)$ and isolated, possibly just touching each other $\left(E_{e l}=0\right)$. Such a situation one can denote as artificially frozen. Only in this case, kinetic and elastic energy vanish and the total energy can remain a constant. If two particles would touch each other with $E_{e l}>0$, at least a part of their elastic energy will be transferred into relative velocity, eventually separating them, so that $E>0$. This motion would eventually lead to more collisions reducing the total energy further. In other words: since no attractive or external forces are involved, there is no reason for a stable overlap or deformation to exist in the long time limit. A similar argumentation for the TC model leads to the analogous conclusion $E_{k}=E_{e}=0$. In all other situations the system evolves with time and the total energy is not constant. The IHS model, in contrast, comes to a halt when the inelastic collapse occurs and the long time limit cannot be reached.

\section{2}

\section{The special case of one bouncing particle}

In order to discuss forces and stresses in the quasi-static limit, we examine the simplest possible case of a ball bouncing on a flat plane under the influence of the gravitational acceleration $g$ pointing in negative $z$ direction. This system is essentially $1 \mathrm{D}$, and in the elastic limit, the particle will bounce forever. Introducing dissipation, with the restitution coefficient $r$, leads to a velocity just after the $n$-th collision $v_{n}^{\prime}=-r v_{n}$ as a function of the velocity $v_{n}$ just before. With the initial velocity $v_{1}$, one has

$v_{n}=r^{n-1} v_{1}$.

The time between the collisions $n$ and $n+1$ is

$t_{n+1}=2 v_{n+1} / g$,

for negative $v_{n+1}$ and negative $g$. In Fig. 2 the vertical position of a bouncing particle is plotted schematically. At each collision energy is lost and the particle stops at time $t=t_{s}$.

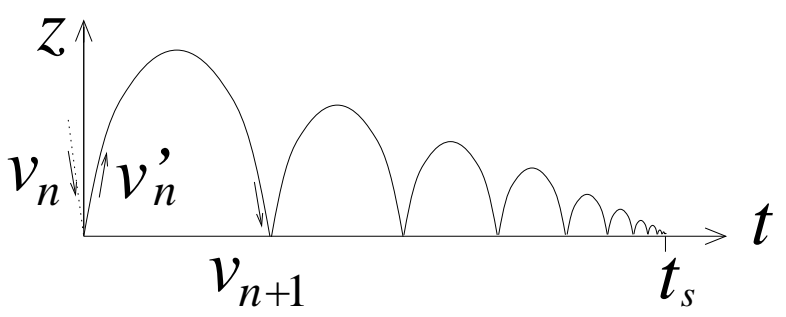

Fig. 2. Trajectory of a bouncing particle on a flat surface as a function of time. At time $t_{s}$ the particle is at rest.

If the particle is infinitely rigid, as assumed by the IHS model, the particle will bounce an infinite number of times before $t_{s}$. At times greater than $t_{s}$, the IHS model is no longer defined. But this picture of an infinitely rigid bouncing ball makes no sense as soon as $t_{n}$ gets comparable to the duration of a contact $t_{c}$. In that case the particle is in steady contact with the plate 30 . Therefore, in the TC model, $r$ is set to 1 when $t_{n}<t_{c}$, and the particle bounces forever on the plate with a constant period which is less than $t_{c}$. This is the TC model's representation of a particle lying on the plate. We now compare $t_{s}$ in the IHS and TC models. Starting with $v_{1} \gg g t_{c} /(2 r)$, the quasi-static limit is reached when $t_{n+1} \leq t_{c}$ so that

$n_{s} \geq \frac{\log \left(\frac{g t_{c}}{2 v_{1}}\right)}{\log r}$

In the hard-sphere limit $n_{s} \rightarrow \infty$ since $t_{c} \rightarrow 0$. The time until the particle has lost all its kinetic energy is

$t_{s}^{(\mathrm{IHS})}=\sum_{n=1}^{\infty} t_{n+1}=\frac{2 v_{1} r}{g} \sum_{n=0}^{\infty} r^{n}=\frac{r}{1-r} t_{1}$

with $t_{1}$ from Eq. (6). The time until a particle with $t_{c}>0$ reaches the quasi-static regime is

$t_{s}^{(\mathrm{TC})}=\sum_{n=1}^{n_{s}} t_{n+1}=\frac{2 v_{1} r}{g} \sum_{n=0}^{n_{s}-1} r^{n}=t_{s}^{(\mathrm{IHS})}\left(1-t_{c} / t_{1}\right)$. 
The difference $\Delta t_{s}$ between these two times is a measure for the difference between a soft particle $\left(t_{c}>0\right)$ and a hard-particle $\left(t_{c}=0\right)$ model:

$\Delta t_{s}=t_{s}^{(\mathrm{IHS})}-t_{s}^{(\mathrm{TC})} \approx \frac{r}{1-r} t_{c}$

The last term in Eq. (10) is obtained by assuming that the time between elastic collisions $n>n_{s}$ is approximately $t_{c}$ (what is almost true for $r \approx 1$ ). Note that $\Delta t_{s}$ will be small, because the contact time is usually small.

\subsection{1}

\section{Performing averages}

In the framework of the TC model, an observable $A$ has to be defined in average over a time-interval $\Delta t$ :

$A(t)=\langle A\rangle=\langle A\rangle_{\Delta t}=\frac{1}{\Delta t} \int_{t-\Delta t}^{t} A\left(t^{\prime}\right) \mathrm{d} t^{\prime}$.

Alternatively, ensemble averages can be performed, however, this option will not be discussed here. The average makes sense only if it averages at least over the duration of a contact $t_{c}$, since the TC model simplifies the reality during times smaller than $t_{c}$. Therefore, averages over longer intervals should be taken to level out the details of the basic assumptions introduced in e.g. Eq. (4).

We now discuss this average, using as an example one particle resting on a flat surface. A real, soft particle resting on the bottom is represented in the TC model as an elastic, hard particle bouncing on the surface with a period $t_{n} \leq t_{c}$. Since it performs a periodic orbit with duration $t_{n}$, one can set $\Delta t=t_{n}$. Thus, integration is performed over one parabola of free flight of the particle. The mean velocity of the bouncing particle is $u=\langle v\rangle=0$, as expected for the quasi-static limit, the mean squared fluctuation velocity is $\left\langle(v-u)^{2}\right\rangle=\left\langle v^{2}\right\rangle=(1 / 12)\left(g t_{n}\right)^{2}$, and the mean separation of the particle from the bottom is $\langle z\rangle=(1 / 12) g t_{n}^{2}$. With these quantities we may identify the energies defined above. The potential energy is connected to the separation from the bottom $E_{p}=$ $m g\langle z\rangle=(1 / 12) m\left(g t_{n}\right)^{2}$, disregarding an additive constant. The total translational energy is $E=E_{k}+E_{e}$, with $E=(m / 2)\left\langle v^{2}\right\rangle=(1 / 24) m\left(g t_{n}\right)^{2}$. Now, we identify the elastic energy with the kinetic energy of the particle(s) which suffered a collision no longer than $t_{c}$ ago. Since the particle collides with a rate $t_{n}^{-1}>t_{c}^{-1}$, all its kinetic energy contributes to $E_{e}$ so that $E_{k}=0$. Note that the values $E_{k}=0, E_{p}>0$, and $E_{e}>0$ correspond to the quasi-static limit discussed above.

In addition, one can calculate the force which the particle exerts onto the bottom as the momentum exchange per unit time $f=\langle\Delta p\rangle=m g$, as to be expected for a particle with mass $m$ in the gravitational field [27].

\subsection{2}

\section{The link to a linear elastic particle}

A soft, elastic particle in contact with the bottom has - in the framework of the simplest linear model [31] - the elastic energy $V(\delta)=(1 / 2) k \delta^{2}$, with stiffness $k$, and overlap or deformation $\delta$. At rest, it exerts the force $f_{k}=k \delta_{0}=$ $m g$ onto the bottom, so that the overlap or deformation is $\delta_{0}=m g / k$. When bouncing, its contact duration is

$t_{c}^{e l}=\pi / \omega=\pi \sqrt{m / k}$,

what leads to the identity $\delta_{0}=g\left(t_{c}^{e l} / \pi\right)^{2}$. The elastic energy of the particle at rest is thus $V\left(\delta_{0}\right)=m\left(g t_{c}^{e l}\right)^{2} /\left(2 \pi^{2}\right)$.

Comparing the soft particle with the TC model from the previous subsection by using either of the relations $\langle z\rangle \equiv \delta_{0}$ or $E_{e} \equiv V\left(\delta_{0}\right)$, leads to

$t_{n} \equiv \sqrt{12} t_{c}^{e l} / \pi$.

From the beginning of this section we remember that the particle reaches its quasi-static limit when $t_{n} \leq t_{c}$. Thus the contact duration $t_{c} \approx t_{n}$ can be identified with the contact duration of the linear soft-sphere model $t_{c}^{e l}$, when disregarding the constant factor $\sqrt{12} / \pi \approx 1$.

\subsection{3}

\section{A Gedanken-Experiment}

In order to clarify the meaning of the different energies calculated above, we assume that we are able to switch off gravity at any time during the period $t_{n}$. A real particle lying on a table with zero kinetic energy will then begin to rise due to the elastic energy stored in the contact. Ideally, for $r=1$, all elastic energy will be transferred to translational motion. Within the framework of the TC model, as discussed here, one can calculate the velocity a particle will eventually reach, after $g$ is set to zero. Since the particle-velocity is phase-dependent, one has to perform the average over all possible phases at which gravity might be switched off. Thus the integration over the period $t_{n}$ has to be split: During the first half-period for $t<t_{n} / 2$, the particle will keep its upwards velocity and move away from the bottom. In the second half-period the particle will suffer one collision with the bottom before it moves upwards. Performing the integration one gets $\left\langle v_{g \rightarrow 0}^{2}\right\rangle=(1 / 24)\left(g t_{n}\right)^{2}(1+r)$. Note that the velocity after switching off gravity, $u_{g \rightarrow 0}$, depends on the time when gravity is switched off. This reflects the fact that a too fine resolution in time uncovers some details of the simplifications in the TC model. However, the elastic case $r \rightarrow 1$ corresponds to the case when all elastic energy $E_{e}$ from the quasi-static regime is transferred into kinetic energy $E_{k}$.

\section{The TC model in elastic systems}

In the following we will mainly focus on the elastic limit $r \rightarrow 1$, and define the properties of interest like the stress tensor, the equation of state, the collision rate, and the elastic energy. For the simulations in this section we use an event driven (ED) simulation method as introduced by Lubachevsky 32. The system has periodic boundaries, and neither walls nor gravity are present. The following discussion concerns only systems in equilibrium and in their center of mass reference frame. Strictly speaking, all collisions in the simulations discussed in this section are "elastic", because $r=1$. However, the TC model distinguishes between those collisions which indicate multiparticle events (where one of the partners has had a collision 
no longer than $t_{c}$ ago), and the rest of the collisions which are truly binary. In this section, only the former type of collisions are called "elastic", for they would also be elastic (in the TC model) when $r \neq 1$. We choose $r=1$ here because this gives systems in equilibrium and allows to obtain good statistics by taking long time averages.

\section{1}

\section{The stress tensor}

The stress tensor, defined for a test-volume $V_{c}$, can be written component-wise as

$\sigma_{\alpha \beta}=\frac{1}{V_{c}}\left[\sum_{j} \ell_{\alpha} f_{\beta}-\sum_{i} m_{i} v_{\alpha} v_{\beta}\right]$.

The first sum runs over all contact points $j$, and the second sum runs over all particles $i$, both within $V_{c}[33,34]$. The indices $\alpha$ and $\beta$ denote the Cartesian coordinates, $\ell_{\alpha}$ are the components of the vector from the center of mass of a particle to its contact point $j$, where a force with components $f_{\beta}$ acts. A particle $i$ has a mass $m_{i}$ and a velocity with the components $v_{\alpha}$.

In the static limit, the second term drops out, since all velocities vanish. In a dilute system without permanent contacts, the first term would be negligible. On the other hand, for a hard-sphere gas, the first term has to be treated differently, since no forces are defined. The dynamic equivalent to $f_{\beta}$ is the change of momentum per unit time $\Delta p_{\beta} / \Delta t$ [31]. For a hard-sphere gas the stress due to collisions may be evaluated as an average over all collisions in the time interval $\Delta t$

$$
\begin{aligned}
& \sigma_{\alpha \beta}(t)=\left\langle\sigma_{\alpha \beta}\right\rangle_{\Delta t}= \\
& \frac{1}{V_{c}}\left[\frac{1}{\Delta t} \sum_{n} \ell_{\alpha}\left(t_{n}\right) \Delta p_{\beta}\left(t_{n}\right)-\sum_{i} m_{i} v_{\alpha}(t) v_{\beta}(t)\right] .
\end{aligned}
$$

Here, the first sum runs over all collisions $n$ occuring in the time between $t-\Delta t$ and $t$. In general, the volume $V_{c}$ and the time-interval $\Delta t$ have to be chosen large enough to allow averages over enough particles and enough collisions, but also small enough to resolve inhomogeneities in space and variations in time. Note that the result may depend on the choice of $\Delta t$ and $V_{c}$ [6], so that this averaging procedure is not necessarily the best choice under all circumstances.

\section{2}

\section{The equation of state}

In order to test the averaged stresses defined above, we compute the mean pressure $P=\left(\sigma_{1}+\sigma_{2}\right) / 2$ from the eigenvalues $\sigma_{1}$ and $\sigma_{2}$ of the stress tensor. The results of simulations with elastic particles, $r=1$, and different volume fractions $\nu$ are compared to the pressure obtained by the two-dimensional equivalent of the Carnahan-Starling formula [3, 29. In Fig. 3, we plot the reduced, dimensionless pressure

$P_{0}-1=\frac{P V}{E}-1=2 \nu g(2 a)$ against the volume fraction $\nu=N \pi a^{2} / V$ 29. We use the pressure $P$, the volume of the system $V$, the total translational energy $E=(1 / 2) \Sigma_{i=1}^{N} m_{i} v_{i}^{2}$, and the particleparticle correlation function evaluated at contact

$g(2 a)=\frac{1-\frac{7}{16} \nu}{(1-\nu)^{2}}$,

taken from Eqs. (28) and (110) in Ref. [3]. The agreement between theory and simulations is perfect since $r=1$, already for a tiny system with $N=42$. The simulations deviate from theory only for volume fractions larger than about 0.7 because then a solid state exists, i.e. the motion of the particles is hindered by their neighbors [35]. The smallest system deviates slightly from the larger ones, indicating finite size effects in the crystalline, high density regime. However, we will not discuss the transition from a fluid, disordered to a 'solid', ordered system here.

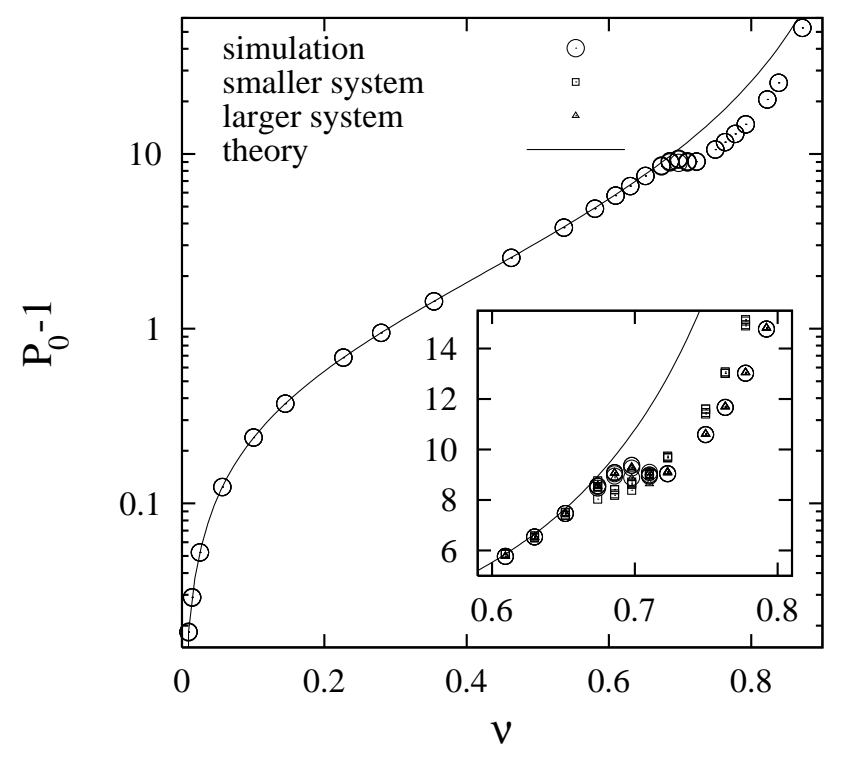

Fig. 3. Reduced dimensionless pressure $P_{0}-1$ plotted against volume fraction $\nu$. Theory (solid line) is compared to simulations (circles) from a system with $N=1435$ particles. In the inset simulations of a smaller and a larger system, with $N=42$ (small squares) and $N=16680$ (small triangles), respectively, are presented in addition. At least three simulations were performed for each $\nu$ value and plotted over each other so that smeared out symbols indicate comparatively large fluctuations at $\nu \approx 0.7$.

\section{3 \\ The collision rate}

The next quantity of interest is the collision rate, i.e. the number of collisions per particle per unit time. Thus, we define the collision rate in the simulations as the inverse of the typical time between contacts

$\mathcal{C}_{r}=t_{n}^{-1}=\frac{2 \mathcal{C}}{N \Delta t}$, 
with $\mathcal{C}=\langle\mathcal{C}\rangle$, the number of collisions per averaging time $\Delta t$. Note that we use $\mathcal{C}$ as the number of collisions within a time $\Delta t$, whereas the total number of collisions since the beginning of the simulation is denoted as $C_{t}$ later on. The prefactor ' 2 ' stems from the fact that each collision involves two particles. In Fig. A we compare the simulation results with the Enskog collision frequency in 2D, as expressed in Ref. [36],

$t_{E}^{-1}=4 a \frac{N}{V} \sqrt{\pi \frac{E}{M}} g(2 a)=\omega_{0}\left(P_{0}-1\right)$

with the particle radius $a$, the total fluctuation kinetic energy $E$, the total mass $M$, and $g(2 a)$ as defined in the previous subsection. From Fig. 1 we observe again a perfect agreement between theory and simulation (circles) for $\nu<0.7$. The difference between Eqs. (16) and (19) is the constant prefactor $\omega_{0}=\sqrt{2 / \pi}\left(v_{T} / a\right)$, with the thermal fluctuation velocity $v_{T}=\sqrt{2 E / M}$. Note that both $E_{k}$ and $E_{e}$ contribute to $E$ and thus to the collision rate. Therefore, $\mathcal{C}_{r}$ can be split into a dynamic and a quasistatic contribution. The latter, the collision rate of elastic collisions is displayed in Fig. 1 in addition to the overall collision rate. In an elastic collision, at least one collision partner had a collision no longer than $t_{c}$ ago, see Eq. (价. Therefore, smaller $t_{c}$ values lead to lower collision rates (the decadic logarithm of $t_{c}=10^{-3} \mathrm{~s}, 10^{-4} \mathrm{~s}, 10^{-5} \mathrm{~s}$, $10^{-6} \mathrm{~s}$, and $10^{-7} \mathrm{~s}$ is given in the inset).

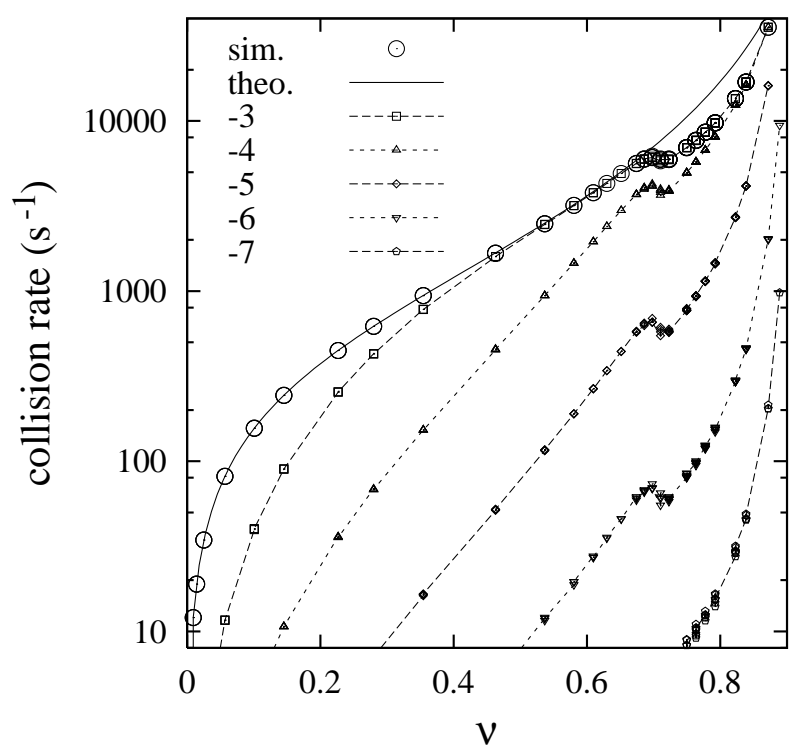

Fig. 4. Collision rates plotted against volume fraction $\nu$ from theory (solid line) and the simulations (circles) from Fig. 3. The dashed lines are inserted between the measured elastic collision rates (small symbols) to guide the eye. The system dependent prefactor of these simulations is $\omega_{0}=656.03 \mathrm{~s}^{-1}$. The small symbols indicate the collision rates of elastic collisions according to the TC model with $\log _{10} t_{c}$ given in the inset.
5.4

\section{Elastic particles, collisions, and energies}

In this subsection, we are interested in $n_{e}=N_{e} / N$, the fraction of particles that are elastic, i.e. which had a collision no longer than $t_{c}$ ago. Furthermore, we want to estimate $c_{e}=\mathcal{C}_{e} / \mathcal{C}$, the fraction of collisions in which elastic particles participate, related to the elastic collision rate in the previous subsection. Finally, following the ideas in subsection 4.1, we will split the total translational energy into a kinetic and an elastic part, i.e. $E=E_{k}+E_{e}$ and determine $e_{e}=E_{e} / E$, the fraction of elastic energy in the system.

First we estimate the probability $p\left(t_{c}\right)=1-N_{e} / N$ that a particle had no collision since a time $t_{c}$ [37. We know that $p(0)=1$, since no particle can suffer a collision in zero time, and $p(\infty)=0$, since any particle will eventually collide (given that $a>0$ and that the system is not artificially frozen). Furthermore, we also know the probability $t_{E}^{-1} \mathrm{~d} t$ that a particle will collide within time $\mathrm{d} t$. Thus we have the probability $1-t_{E}^{-1} \mathrm{~d} t$ that the particle will not collide. Multiplying the probability that it did not collide until $t_{c}$ with $1-t_{E}^{-1} \mathrm{~d} t$ finally gives the probability that it does not collide until $t_{c}+\mathrm{d} t$. First order Taylor expansion of $p\left(t_{c}+\mathrm{d} t\right)=p\left(t_{c}\right)\left(1-t_{E}^{-1} \mathrm{~d} t\right)$ around $t_{c}$ leads to $\frac{\mathrm{d}}{\mathrm{d} t} p(t)=p(t) t_{E}^{-1}$. Integration for constant collision frequency $t_{E}^{-1}$ gives the fraction of 'elastic' particles

$n_{e}=1-p\left(t_{c}\right)=1-\exp \left(-t_{c} t_{E}^{-1}\right)$.

In Fig. 司 we present the quality factor $q_{n}=\left\langle N_{e}\right\rangle /\left(N n_{e}\right)$, the ratio of the measured fraction of elastic particles and of the analytical expression from Eq. (20). Each point corresponds to an average over 30 snapshots from simulations with $N=1435$ particles.

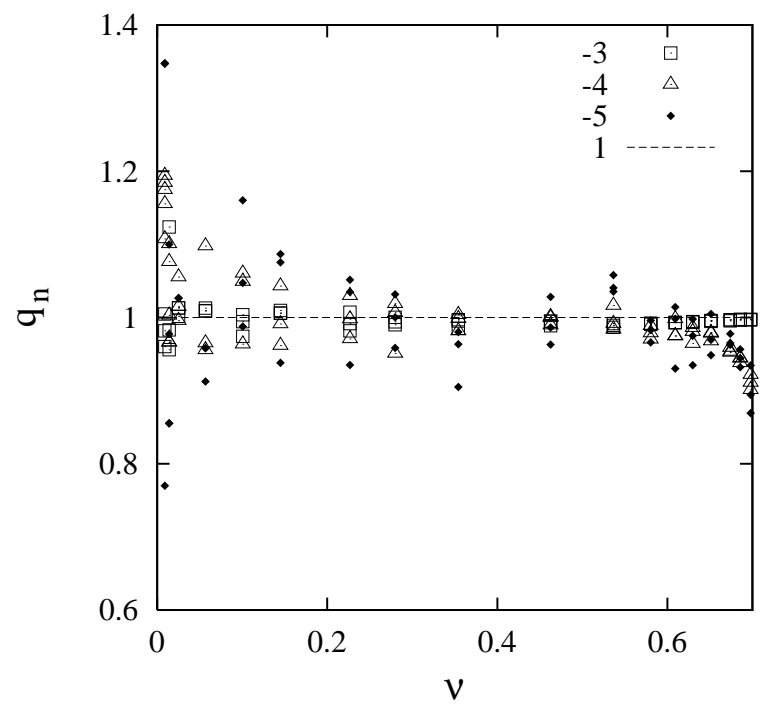

Fig. 5. The quality factor $q_{n}$, i.e. the ratio of $\left\langle N_{e}\right\rangle / N$ and $n_{e}$ from Eq. (20) plotted against $\nu$. The simulations are the same as in Fig. 3. Values close to unity indicate good agreement between theory and simulations for the $t_{c}$ values used. To distinguish between different $t_{c}$ values, $\log _{10} t_{c}$ is given in inset. 
Data for different $t_{c}$ values can be obtained from the same simulation since each particle 'remembers' when it had its last collision at time $t_{n}^{(i)}$ and because no dissipation is active. Dissipation would make the sequence of collisions dependent on $t_{c}$. The agreement between simulations and the theoretical prediction, Eq. (20), is reasonable $\left(q_{n} \approx 1\right)$. Deviations occur for small volume fractions $\nu$ due to bad statistics and for large volume fractions $\nu>0.7$ due to the transition to the ordered system.

However, Eq. (20) is not the fraction of elastic collisions $c_{e}$ which instead has to be computed as the sum of probabilities that either both or only one of the collision partners belongs to the elastic particles. Thus one gets

$c_{e}=n_{e}^{2}+2 n_{e}\left(1-n_{e}\right)=1-\exp \left(-2 t_{c} t_{E}^{-1}\right)$,

almost in agreement with the simulations as displayed in Fig. 6. The quality factor $q_{c}=\left\langle\mathcal{C}_{e}\right\rangle /\left(\langle\mathcal{C}\rangle c_{e}\right)$ plotted against $\nu$ indicates a difference between the measured and the calculated fraction of elastic collisions. Interestingly, the simulation data show that there occur about five percent less elastic collisions than expected from Eq. (21). The data presented in Fig. 6 were obtained by averaging $\left\langle\mathcal{C}_{e}\right\rangle /\langle\mathcal{C}\rangle$ over a time interval $\Delta t$ large enough to allow for more than about $10^{4}$ collisions per particle. A more rigorus theoretical treatment that would lead to the exact value of $c_{e}$ is beyond the scope of this study and will be discussed elsewhere [38].

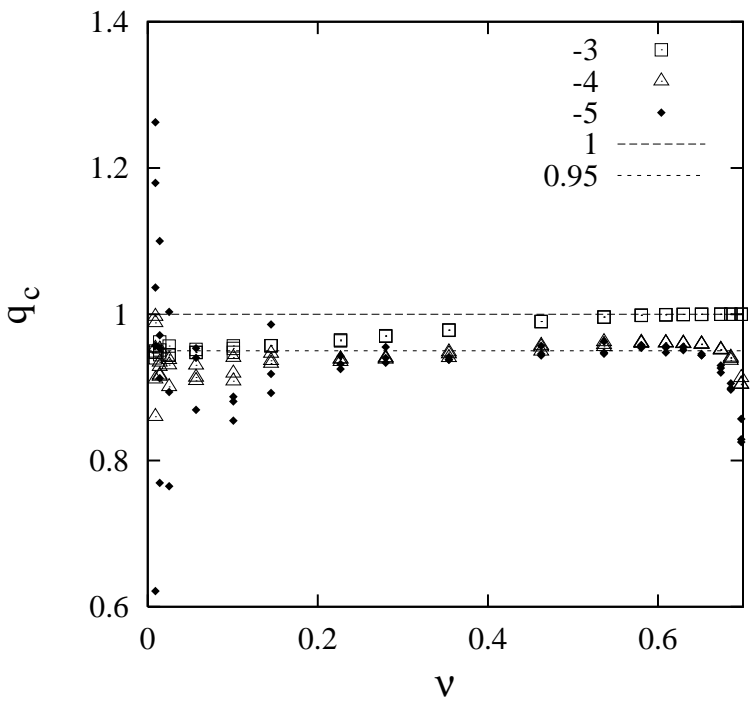

Fig. 6. The ratio of simulational and theoretical results on the probability for elastic collisions plotted against $\nu$. Exact agreement would correspond to $q_{c}=1$.

Finally, we estimate the elastic energy contained in the system in a simple minded, mean-field way. The fraction of elastic energy $e_{e}$ should be the product of $n_{e}$ and $E$ when all particles would have the same (mean) energy and would contribute to $E_{e}$ with the same probability. Unfortunately, the simulation results in Fig. 1 indicate that the mean-field approach is not valid. The discrepancy between the mean-field estimate presented above for $n_{e}$ and the numerical simulations can, however, be understood via qualitative arguments. Particles with greater velocities have a higher probability to collide and, therefore, have a greater collision rate than slower particles. Due to the greater collision rate, fast particles are more likely to contribute to $E_{e}$ and $E_{e}$ is increased since faster particles contribute with a greater energy 38 .

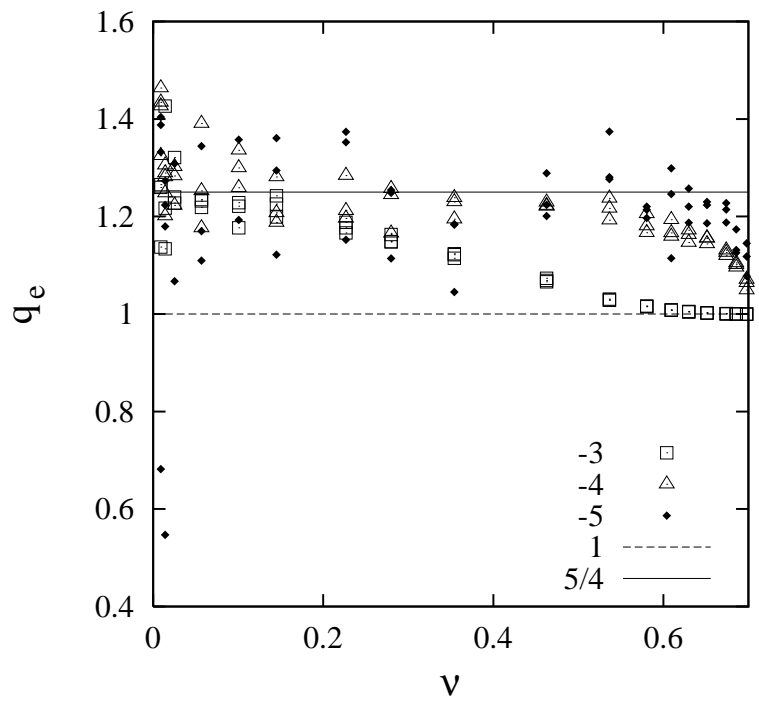

Fig. 7. The ratio of simulational and theoretical results on the fraction of elastic energy in the system plotted against $\nu$. A quality factor $q_{e}=1$ would correspond to exact agreement. The value $5 / 4$ was taken from a more elaborate kinetic theory calculation 38.

\section{6}

\section{The TC model in dissipative systems}

One of the phenomena which received a great deal of attention in the last years is the clustering instability in rather dilute systems of dissipative particles [12, 39, 40]. Initially given a homogeneous density and a Maxwellian velocity-distribution, the system cools due to dissipation [12, 36. This cooling regime is unstable to perturbations with large enough wavelength, i.e. small enough wavenumber 12, 41. The homogeneous state can be well described by hydrodynamic theory, but the standard description breaks down as soon as the perturbations grow - assumptions like homogeneity or "molecular chaos" are not longer true [28, 40]. Furthermore, it has been recognized that a hydrodynamic description breaks down due to the divergence of the collision rate and the connected dramatic decrease of free volume during the inelastic collapse [42, 43]. The instability may be understood in a qualitative manner: The homogeneous state contains thermal velocity fluctuations. A convergent velocity fluctuation leads to increasing densities in certain regions. As the collision rate increases in these regions, so does the energy dissipation rate. If the energy dissipation rate is great enough, the pressure cannot reverse the convergent flow. If this process 
is not terminated by sufficiently strong perturbations, it is self-stabilizing, causes clusters, and may eventually lead to the inelastic collapse.

The clustering instability and the inelastic collapse were carefully examined in $1 \mathrm{D}$ [8, 8, 19, 44, 47] and in 2D [9, $12,20,39,48,51$. Cluster growth could be described theoretically in the case of irreversible aggregation $(r=0)$ [49, 52] and, more recently, also a theory for the growth of density fluctuations was proposed for $r \neq 0$ [53. Detailed examination of the inelastic collapse by McNamara and Young [12] led to the picture of different 'phases'. In a periodic system without external forcing exists a critical dissipation - connected to system size, volume fraction and restitution coefficient - above which clustering occurs and below which the system stays in molecular chaos. In the transient regime shearing modes or large scale eddies are frequently observed. The case of homogeneous cooling is rather well understood 28 so that we mainly focus on situations with rather strong dissipation when the system is no longer homogeneous.

Using event-driven simulations, periodic 2D systems with side-length $L=l /(2 a)$ in $2 \mathrm{D}$ are examined in the following. A system contains $N$ particles of radius $a$ and volume fraction $\nu=N \pi(a / l)^{2}=(\pi / 4) N / L^{2}$. In this section the particles are dissipative with a restitution coefficient $r$. We apply the TC model as described above, i.e. we use Eq. (4) with the parameter $t_{c}$ to be specified. Initially we arrange the particles on an ordered lattice and give each of them a random velocity; then the system is equilibrated with $r=1$ until a Maxwellian velocity distribution is obtained. Finally, dissipation is switched on and the simulation starts at $t=0 \mathrm{~s}$.

\section{1}

\section{Inhomogeneous cooling - Finite size effects}

In this subsection we discuss typical simulations with volume fraction $\nu=0.227$, restitution coefficient $r=0.40$, and contact duration $t_{c}=10^{-5} \mathrm{~s}$. The system size is varied so that $N=97776,22960,5740,1435,378$, and 42 particles fit into the system. In Fig. 8, the dimensionless kinetic energy $T=E(t) / E(0)$ is plotted against the rescaled time $\tau=t_{E}^{-1}(0) t$, with the initial collision rate $t_{E}^{-1}(0) \approx 444 \mathrm{~s}^{-1}$ for all simulations.

Following the estimate of a critical restitution coefficient, see Eq. (9) in Ref. [12], below which the inelastic collapse can be expected, we compute for our simulations

$r_{c}(N) \approx \tan ^{2}\left[\frac{\pi}{4}\left(1-\frac{1}{\lambda_{\mathrm{opt}}}\right)\right]$,

with the non-dimensional optical depth $\lambda_{\text {opt }}=\sqrt{\pi N \nu} / 2$. Comparing the critical restitution coefficients $r_{c}(97776)=$ $0.976, r_{c}(22960)=0.952, r_{c}(5740)=0.906, r_{c}(1435)=$ $0.821, r_{c}(378)=0.680$ and $r_{c}(42)=0.296$ to $r=0.40$ used for the simulations, we note that all are larger, $r_{c}>r$, except for the tiny system with $N=42$. However, due to the TC model used in our simulation no collapse is observed. The dashed line in Fig. 8 gives the analytical solution for

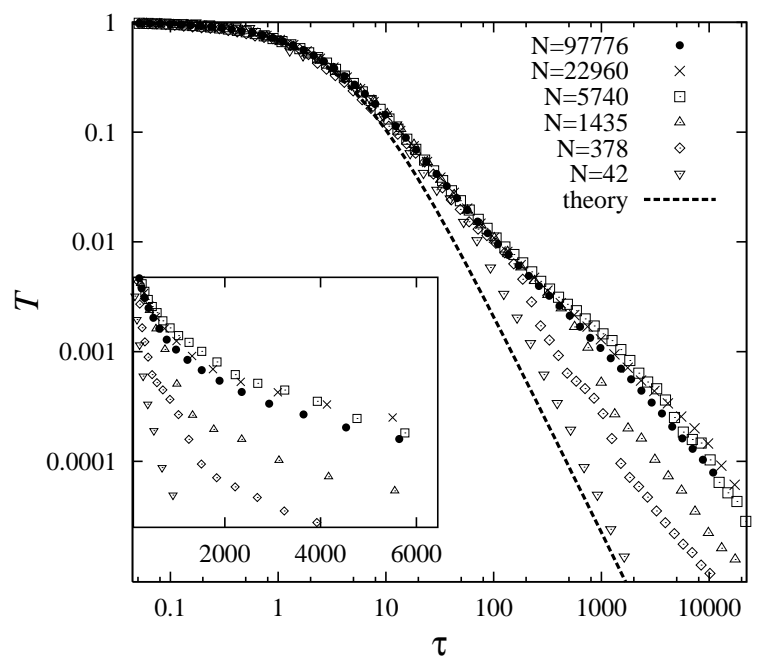

Fig. 8. $T$ as function of $\tau$ for simulations with $N=97776$, 22960, 5740, 1435, 378, and 42, $\nu=0.227, r=0.4$, and $t_{c}=$ $10^{-5} \mathrm{~s}$. The dashed line gives the solution of the homogeneous cooling state Eq. (23).

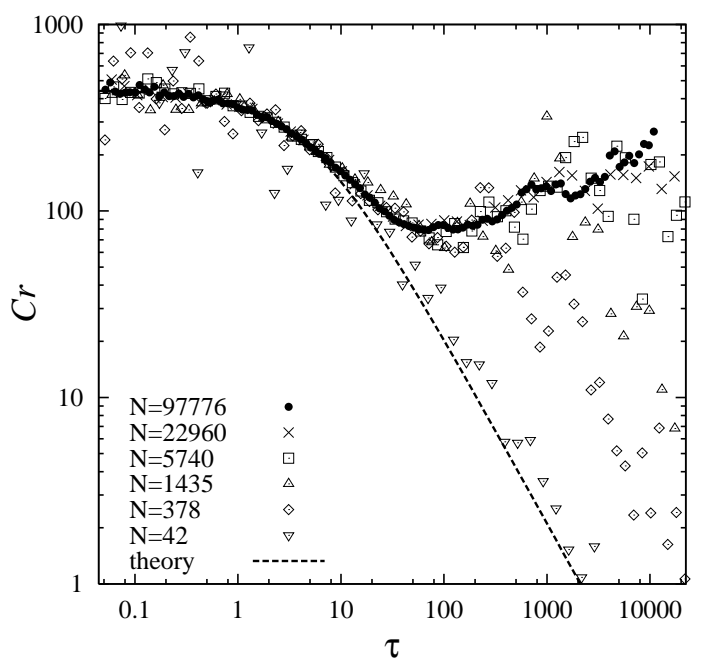

Fig. 9. Collision rate $\mathcal{C}_{r}$, see Eq. (18), as function of $\tau$ from the same simulations as in Fig. The dashed line gives the solution of the homogeneous cooling state.

the homogeneous cooling state (HCS) of smooth particles (see [36] and references therein):

$T=\left(1+\frac{1-r^{2}}{4} \tau\right)^{-2}$.

Only the smallest system is close to the theoretical prediction, all others deviate stronger with increasing system size.

The collision rate in the homogeneous cooling state is directly linked to the fluctuation velocity $v_{T}$, see Eq. 19 . Because $v_{T}$ is the only quantity that changes during the simulations, we can also express the collision rate in terms of $\sqrt{T}=v_{T} / v_{0}$ so that due to normalization $T_{0}=1$, one has $\mathcal{C}_{r}=\mathcal{C}_{r}(0) \sqrt{T}$. In order to test this prediction, we plot $\mathcal{C}_{r}$ against $\tau$ in Fig. 9 from the same simulations as in Fig. 8 . 

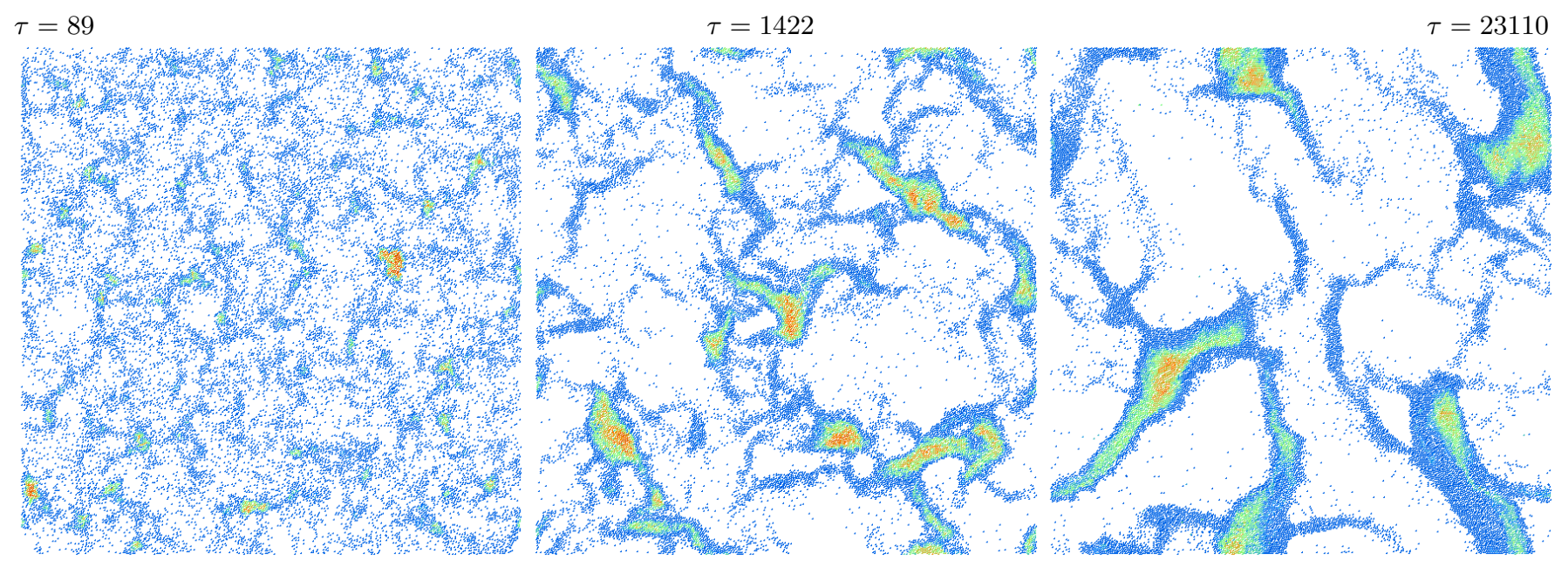

Fig. 10. ED simulation with $N=22960$ particles in a system of size $L=280$, volume fraction $\nu=0.227$, restitution coefficient $r=0.4$, and contact duration $t_{c}=10^{-5} \mathrm{~s}$. The collision rate is color-coded red $\left(\mathcal{C}>1100 \mathrm{~s}^{-1}\right)$, green $\left(\mathcal{C} \approx 700 \mathrm{~s}^{-1}\right)$, and blue $\left(\mathcal{C}<300 \mathrm{~s}^{-1}\right)$.
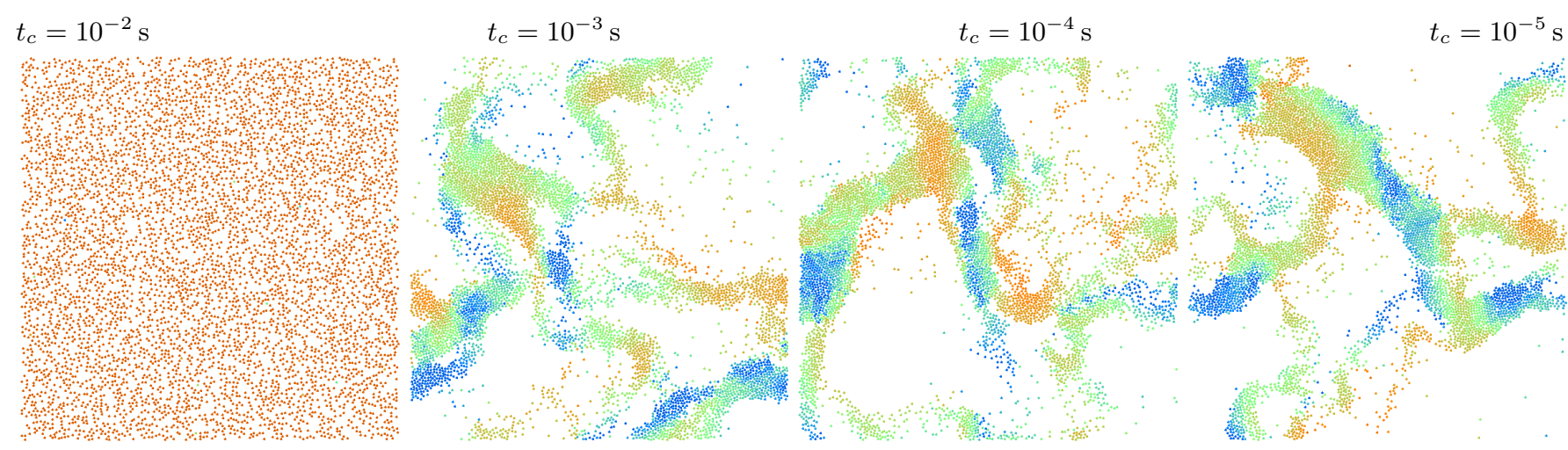

$$
t_{c}=10^{-6} \mathrm{~s}
$$

$$
t_{c}=10^{-8} \mathrm{~s}
$$

$$
t_{c}=10^{-10} \mathrm{~s}
$$

$$
t_{c}=10^{-12} \mathrm{~s}
$$

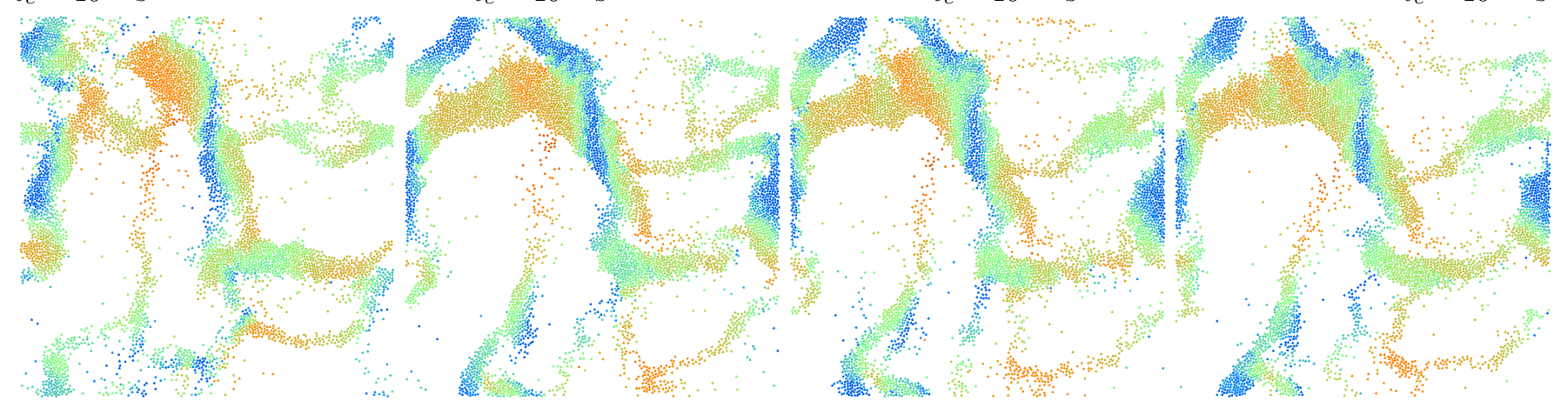

Fig. 11. Snapshots at time $t=5 \mathrm{~s}$, i.e. $\tau=2222$, from ED simulations started from identical initial conditions, with $N=5740$, $L=140, \nu=0.227, r=0.4$, and different contact duration $t_{c}$. The mean fluctuation velocity (relative to the center of mass) is color-coded red $\left(v_{T} / v_{0}>0.045\right)$, green $\left(v_{T} / v_{0} \approx 0.02\right)$, and blue $\left(v_{T} / v_{0}<0.01\right)$, with initial mean velocity $v_{0}=v_{T}(0)=$ $0.3615 \mathrm{~m} \mathrm{~s}^{-1}$.

As can be seen in both figures 8 and 9 , for small $\tau<5$ the simulations agree with the theory. For larger $\tau$ deviations from the homogeneous cooling regime occur and the energy decay slows down due to the density instability and the build-up of clusters. The behavior of the energy as a function of time is independent of the system size up to $\tau \approx 200$ when the small system $N=378$ starts to deviate from the larger systems. The deviation from the common behavior occurs later with increasing system size, indicating finite-size effects. A closer examination of snapshots from the simulations leads to the conclusion that the deviation from the slow cooling regime occurs when the clusters have reached the system size. The tiny system $N=42$ is anyway too small to allow large scale structures and therefore follows the HCS prediction more closely. 
In Fig. 10 snapshots of the simulation with $N=22960$ from Fig. 8 are displayed. With increasing time $\tau$ structures build up in the system and grow in size. In the red and green regions in the centers of the clusters the collision rate is largest. The size of the clusters grows with time and the qualitative cooling behavior of the system changes as soon as the clusters get to be as large as the system.

\section{2}

\section{Variation of the contact duration}

In the following set of simulations, $t_{c}$ is varied while the other parameters are fixed to $N=5740, \nu \approx 0.227$, and $r=0.4$. In Fig. 11 snapshots from the simulations with different contact duration are displayed. The simulations were set up with identical initial conditions and the snapshots were taken at the same time. For $t_{c}=10^{-2} \mathrm{~s}$ cooling is almost hindered, since the time between collisions is much greater than the dissipation cut-off $t_{c}$. The simulations with $t_{c} \leq 10^{-3} \mathrm{~s}$ all show density instabilities and clusters, however, the pictures differ in details. The change in behavior between $t_{c}=10^{-2} \mathrm{~s}$ and $10^{-3} \mathrm{~s}$ will be dicussed in more detail elsewhere [38]. Only for very small $t_{c} \leq 10^{-8} \mathrm{~s}$ the simulations lead to almost identical results. The color of the snapshots represents the particle velocities in the center of mass reference frame. A large cluster with different colors is thus not a block without internal motion but rather a liquid-like arrangement with strong internal shearing.

In Fig. 12, the dimensionless kinetic energy $T$ is plotted against the rescaled time $\tau=t_{E}^{-1}(0) t$, with the initial collision rate $t_{E}^{-1}(0) \approx 444 \mathrm{~s}^{-1}$. As contact duration, various values between $t_{c}=10^{-2} \mathrm{~s}$, and $10^{-12} \mathrm{~s}$ were used $\left(\log _{10} t_{c}\right.$ is given in the inset for identification). The simulations with $t_{c}<5 \times 10^{-4}$ s lead to the same functional behavior of $T$ as function of $\tau$, except for small deviations for large $\tau$, as can be obtained from the inset. However, the data with $t_{c} \leq 10^{-6} \mathrm{~s}$ cannot be distinguished, i.e. for small enough $t_{c}$ the energy of the system is not influenced by the contact duration. For very small $\tau<5$, the simulations agree with the theoretical result for the homogeneous cooling state from Eq. (23). For larger $\tau$ deviations from the homogeneous cooling regime occur and the energy decay slows down due to the density instability and the build-up of clusters.

The simulations with the largest $t_{c}$ cool slowest since the number of elastic collisions increases with $t_{c}$, see Eq. (21). Inserting $t_{c}=10^{-3} \mathrm{~s}$ and $t_{E}^{-1}(0)$ into Eq. (21), which was derived for elastic system in equilibrium however, leads to $c_{e} \approx 0.59$, whereas the contact duration of $t_{c}=$ $10^{-4} \mathrm{~s}$ leads to a much smaller fraction of elastic contatcs $c_{e} \approx 0.085$. Evidently, dissipation is almost inactive when $t_{c}$ is as large as $t_{c}=10^{-2}$ s so that $c_{e} \approx 0.9999$. On the other hand, the effect of $t_{c}$ will be negligible for $t_{c}<10^{-5} \mathrm{~s}$ when the fraction of elastic collisions vanishes $c_{e}<9 \times 10^{-4}$. In summary, the contact duration $t_{c}$ slows down dissipation in the system - the larger $t_{c}$ the stronger the effect. For small enough $t_{c}$ the system is not affected and the variation of the energy $T$ with the contact duration $t_{c}$ is rather weak. Note that other quantities, as

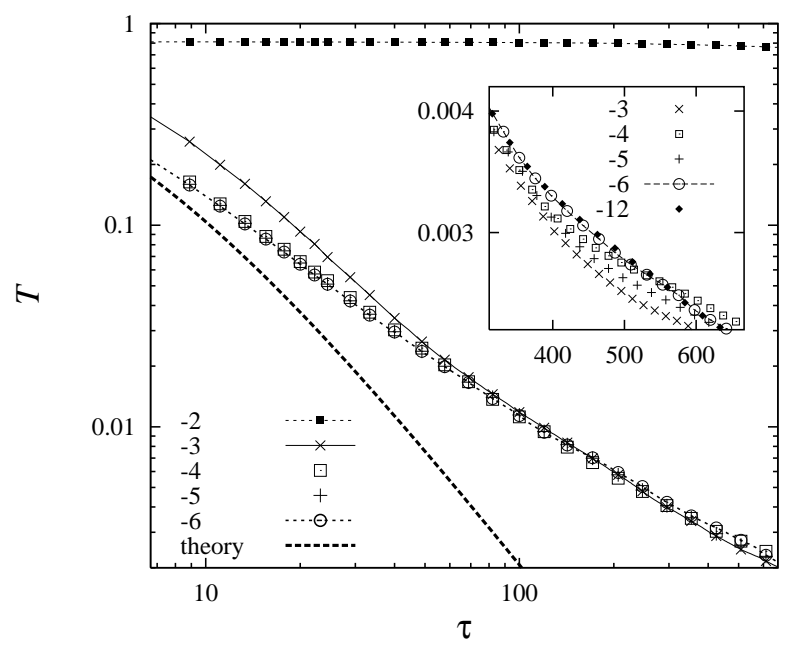

Fig. 12. Dimensionless energy $T$ plotted against $\tau$ from simulations with $r=0.4$ and $\log _{10} t_{c}$ as given in the plot. The inset is a zoom into the plot at large $\tau$ and small $T$. The dashed line corresponds to Eq. (23) with $r=0.4$.

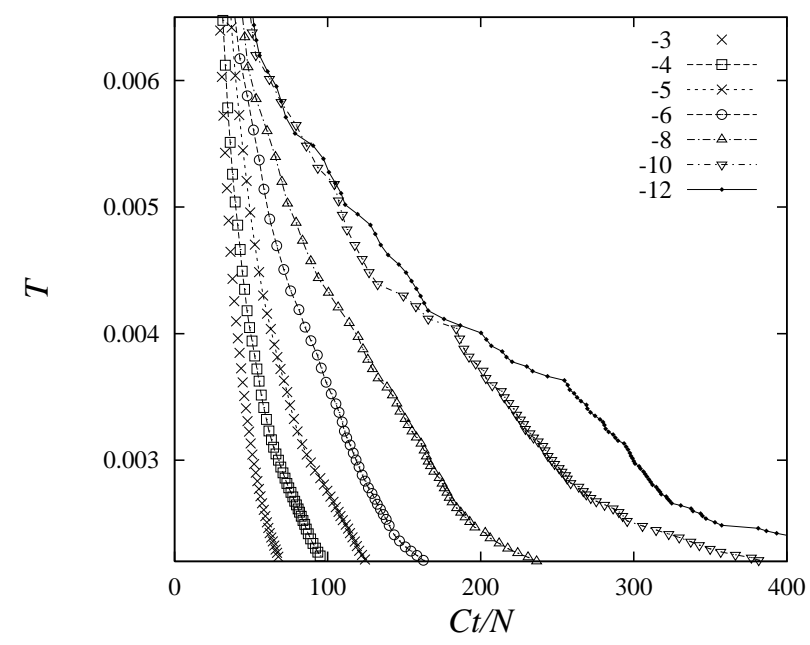

Fig. 13. $T$ plotted against $C_{t} / N$ for the same simulations as in Fig. 12 .

e.g. the total number of collisions per particle $C_{t} / N$, can vary strongly with $t_{c}$, as evidenced in Fig. 13. The smaller $t_{c}$ the more collisions occur in the system during a fixed time interval. Sometimes, for $t_{c} \leq 10^{-10} \mathrm{~s}$, jumps in $C_{t} / N$ are observed. As a consequence, the total number of collisions looses its meaning as a system-inherent time scale as soon as the system becomes inhomogeneous. This can also be understood when recalling that an inhomogeneous system is a system that has non-constant density, fluctuation velocity, pressure, and collision rate. Different parts of the system, evolving with different collision rates, cannot be assumed to follow a common time-scale. On the other hand, the variation of $C_{t} / N$ with $t_{c}$ allows to see the TC model also as a way to reduce the computational effort by decreasing the global number of events that have to be handled, however, without affecting physical observables like $T$. 
(a)

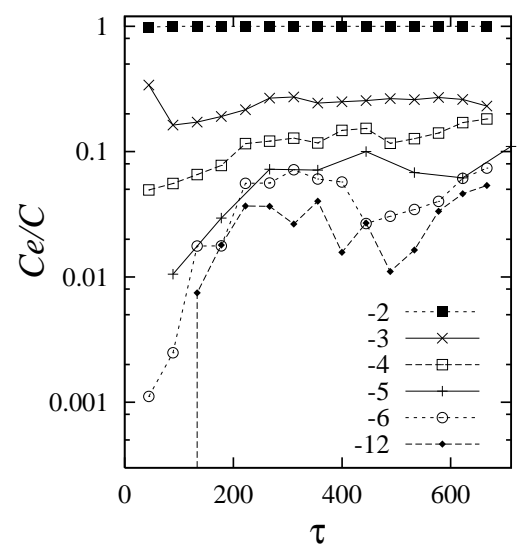

(b)

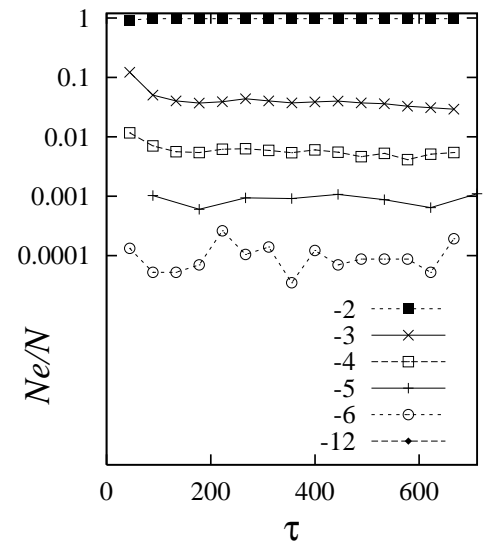

(c)

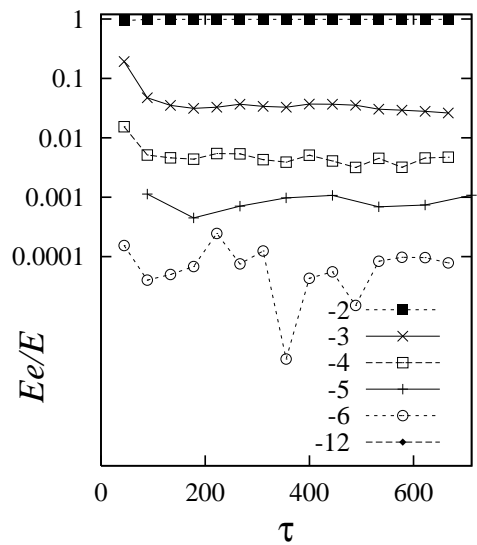

Fig. 14. (a) The fraction of elastic collisions $\mathcal{C}_{e} / \mathcal{C}$ as function of $\tau$ with $r=0.4$ and $\log _{10} t_{c}$ as given in the inset. The simulations are the same as in Fig. 12, however, each point represents an average over all collisions in the time interval $\Delta t=0.1 \mathrm{~s}$. (b) The fraction of elastic particles $N_{e} / N$ plotted against $\tau$ for the same simulations as in (a). Each data point represents an average over 20 snapshots in the time interval $\Delta t=0.1 \mathrm{~s}$. Note the different vertical axis scaling in (a) and (b). (c) The fraction of elastic energy $E_{e} / E$ plotted against $\tau$ for the same simulations and the same averaging procedure as in (b).

Another way to report the effect of the TC model on the simulation results is to take a closer look at the fractions of elastic collisions, particles, and energy in the system. In Fig. 14(a), (b), and (c) these quantities are displayed respectively. The fraction of elastic collisions $c_{e}=\mathcal{C}_{e} / \mathcal{C}$ is systematically larger than both the fraction of elastic particles $n_{e}=N_{e} / N$ and the fraction of elastic energy in the system $e_{e}=E_{e} / E$. The latter two quantities are always comparable, whereas $c_{e}$ depends on $t_{c}$ in a different way. The fraction of elastic collisions is correlated to $t_{c}$, at the beginning of the simulation, as long as the system is homogeneous. For larger $\tau$ the fraction of elastic collisions fluctuates around at a finite value, independent how small $t_{c}$ is. Even the simulation with $t_{c}=10^{-12} \mathrm{~s}$ has a rather large fraction of elastic collisions. However, setting a large $\mathcal{C}_{e}$ in relation to the large total number of collisions $C_{t} / N$, or equivalently $\mathcal{C}_{r}$, leads to the conclusion that the TC model adjusts $c_{e}$ to an finite, almost constant value. In contrast, the elastic energy and the fraction of elastic particles decrease systematically with $t_{c}$. These two quantities are directly affected by $t_{c}$. Concerning averages we must remark that the procedure to obtain $\mathcal{C}$ is different from the one used to obtain average particle numbers or energies. While all collisions in the averaging time interval are summed up to $\mathcal{C}$, only a certain number of snapshots is evaluated to compute $\left\langle N_{e}\right\rangle$ and $\left\langle E_{e}\right\rangle$.

\section{3}

\section{Variation of the restitution coefficient}

For the parameter study in this subsection, the system with $N=5740, \nu=0.227$, and a fixed contact duration $t_{c}=10^{-5} \mathrm{~s}$ is used. This choice of $t_{c}$ is arbitrary, however, as we have shown in the previous subsection, it is a reasonable choice in the framework of the TC model: $t_{c}$ has a negligible effect on $T$. Furthermore, such a $t_{c}$ value is of the same order of magnitude as the contact duration of e.g. two steel spheres with radius $a=1.5 \mathrm{~mm} 15$.

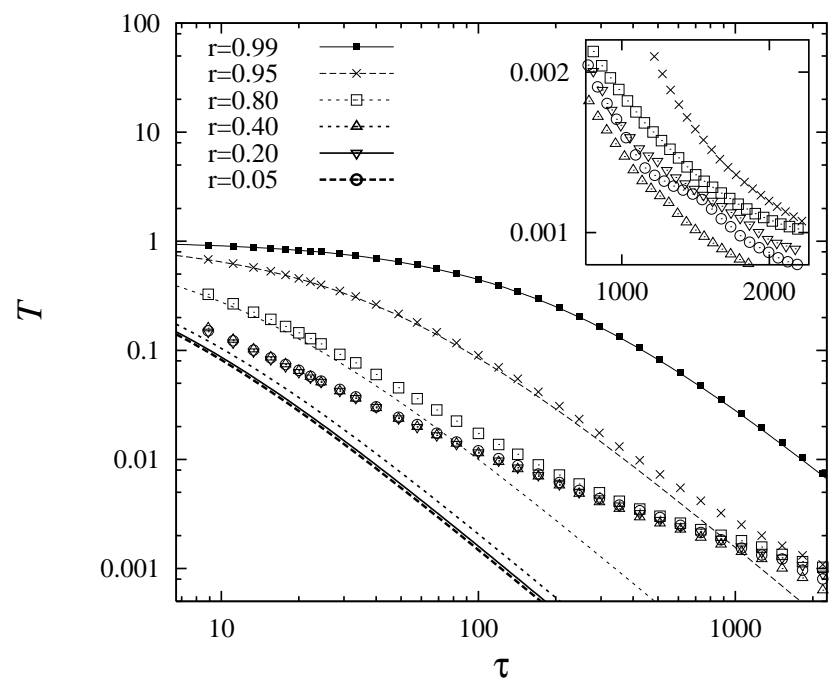

Fig. 15. Dimensionless energy $T$ plotted against $\tau$ from simulations with $t_{c}=10^{-5} \mathrm{~s}$ and $r$ as given in the plot. The inset is a zoom into the plot at large $\tau$ and small $T$. The points are simulation data, the lines give Eq. (23) for the corresponding $r$ values.

In Fig. 15, the dimensionless kinetic energy $T$ is again plotted against the rescaled time $\tau$, with the initial collision rate $t_{E}^{-1}(0) \approx 444 \mathrm{~s}^{-1}$. Simulations are performed for different restitution coefficients as given in the plot, and are compared to the analytical solution of the homogeneous cooling state, see Eq. (23). Only for $r=0.99$, we evidence reasonable agreement with the theory. With decreasing $r$, deviations from the theoretical curve occur already for smaller and smaller $\tau$. However, for $r \leq 0.4$, there are only small differences between simulations with different $r$, as can be seen more clearly in the inset. 
(a)

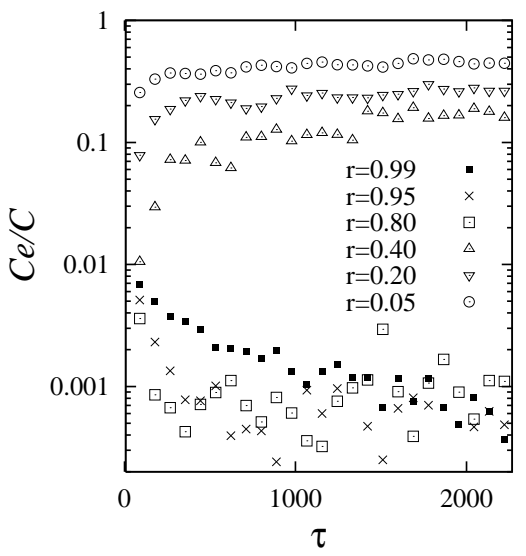

(b)

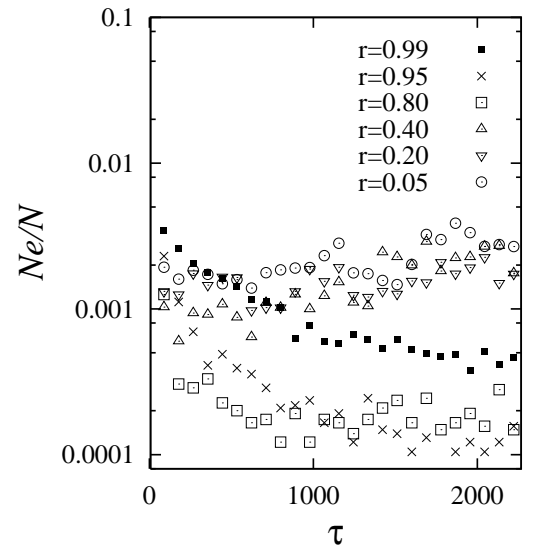

(c)

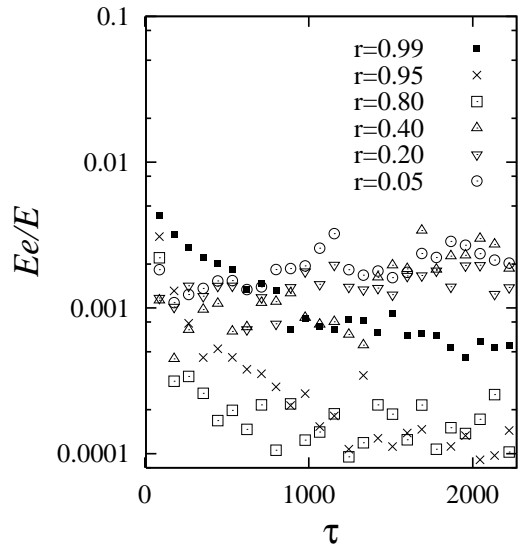

Fig. 16. (a) The fraction of elastic collisions $\mathcal{C}_{e} / \mathcal{C}$ as function of $\tau$ with $t_{c}=10^{-5} \mathrm{~s}$ and $r$ as given in the inset. The simulations are the same as in Fig. 15, however, each point represents an average over all collisions in the time interval $\Delta t=0.2 \mathrm{~s}$. (b) The fraction of elastic particles $N_{e} / N$ plotted against $\tau$ for the same simulations as in (a). Each data point represents an average over 20 snapshots in the time interval $\Delta t=0.2 \mathrm{~s}$. Note the different vertical axis scaling in (a) and (b). (c) The fraction of elastic energy $E_{e} / E$ plotted against $\tau$ for the same simulations and the same averaging procedure as in (b).

We do not present a plot of $T$ against $C_{t} / N$ here, but make some qualitative remarks on the total number of collisions. We evidence that the total number of collisions per particle varies with $r$ as it varies with $t_{c}$. For large $r$ and small $C_{t} / N$ the energy behaves as $T=$ $\exp \left[-\frac{1}{2}\left(1-r^{2}\right) C_{t} / N\right]$, as can be simply derived using Eq. (23) and integrating the collision rate $\mathcal{C}_{r}$ over $\tau$. This behavior is obtained only for $r \approx 1$, already for $r \leq 0.95$ the simulations deviate from the theoretical prediction.

For strong dissipation, only a few particles perform many collisions. One can expect that the time between the collisions of such particles drops below the threshold $t_{c}$ so that $r$ is set to unity according to Eq. (4). The TC model is active, hinders dissipation, and thus evades the inelastic collapse. The number of particles affected by the TC model will be discussed in the following. In Fig. 16 we present data on $c_{e}, n_{e}$, and $e_{e}$ in a way similar to Fig. 14. Again, we obtain that $c_{e}$ behaves differently from $n_{e}$ and $e_{e}$. All simulations with $r \geq 0.80$ have a negligible fraction of elastic collisions $\left(c_{e}<2 \times 10^{-3}\right.$ for long times). The simulations with stronger dissipation $(r<0.80)$ have an increasing number of elastic collisions with increasing dissipation, i.e. decreasing $r$.

From the data on $N_{e}$, we can estimate the number of particles with the largest collision rate, which typically are involved into elastic collisons. A fraction of $n_{e}=0.002$ corresponds in the case of $N=5740$ to $N_{e} \approx 10$ particles. Even in the case of very strong dissipation only about 10 particles are affected by the TC model.

\section{7}

\section{Conclusion and Outlook}

In this study we discussed the TC model, an extension of the frequently used inelastic hard-sphere model. Introducing the contact duration $t_{c}$ as a material parameter, multiparticle interactions are defined in some sense. They concern particles with large collision rates which are assumed to contribute to the elastic energy in the system (which cannot be dissipated). The TC model can reach a quasistatic situation when only elastic and potential energy remain. Dissipation is locally inactive for large collision rates, i.e. the elastic limit where multiparticle contacts occur, and active for rare events, i.e. the dissipative limit where contacts are binary almost always. The TC model allows simulations in ranges of parameter space, where the classical inelastic hard-sphere model breaks down due to the inelastic collapse. Potential, kinetic, and elastic energies as well as stresses and forces are defined as averages over time-intervals comparable to $t_{c}$. The material parameter $t_{c}$ can be identified with the contact duration $t_{c}^{e l}$ of a simple linear particle model, involving e.g. particle mass and stiffness.

Furthermore, mean-field estimates for the fraction of elastic particles $n_{e}$, the fraction of elastic collisions $c_{e}$, and the fraction of elastic energy $e_{e}$ in the system are presented. The simulation results indicate that a more elaborate theory is required to explain the obtained discrepancies. However, the definition of $n_{e}, c_{e}$, and $e_{e}$ is also valid in non-equilibrium, dissipative systems. Detailed examinations of the inhomogeneously cooling situation leads to the conclusion that the TC model affects a small fraction of the particles only - just as the inelastic collapse. Therefore, we beleive that the TC model removes inelastic collapse in a physically reasonable way, without disturbing the global behavior of the system, i.e. the clustering. This is strictly true for realistically small $t_{c}$ values, because an extremely large $t_{c}$ changes the global behavior dramatically.

The TC model is defined for arbitrary dimension so that an extension to three dimensional systems is straightforwardly performed [36], and also gravity or moving walls can be implemented [14, 27] without loosing its generality. A future aim is to include the TC model into kinetic theories in the style of Haff [2] where it will affect only the energy dissipation rate via a correction factor $1-c_{e}$. 
However, besides excluded volume and dissipation, one important property of granular materials, namely friction, is missing in its present form. A proper definition of friction in the framework of the TC model is in progress and has to be tested with systems like particles on an inclined plane or sandpiles which are kept at rest by friction.

\section{References}

1. Physics of dry granular media - NATO ASI Series E 350, edited by H. J. Herrmann, J.-P. Hovi, and S. Luding (Kluwer Academic Publishers, Dordrecht, 1998).

2. P. K. Haff, J. Fluid Mech. 134, 401 (1983).

3. J. T. Jenkins and M. W. Richman, Phys. of Fluids 28, 3485 (1985).

4. C. K. K. Lun and S. B. Savage, Acta Mechanica 63, 15 (1986).

5. A. Goldshtein and M. Shapiro, J. Fluid Mech. 282, 75 (1995).

6. I. Goldhirsch, in Physics of dry granular media - NATO ASI Series, edited by H. J. Herrmann, J.-P. Hovi, and S. Luding (Kluwer Academic Publishers, Dordrecht, 1998), p. 371.

7. B. Bernu and R. Mazighi, J. Phys. A: Math. Gen. 23, 5745 (1990).

8. S. McNamara and W. R. Young, Phys. Fluids A 4, 496 (1992).

9. S. McNamara and W. R. Young, Phys. Rev. E 50, R28 (1994).

10. D. Goldman et al., Phys. Rev. E 57, R4831 (1998).

11. S. E. Esipov and T. Pöschel, J. Stat. Phys. 86, 1385 (1997).

12. S. McNamara and W. R. Young, Phys. Rev. E 53, 5089 (1996).

13. E. L. Grossman, Phys. Rev. E 56, 3290 (1997).

14. S. Luding, E. Clément, J. Rajchenbach, and J. Duran, Europhys. Lett. 36, 247 (1996).

15. S. Luding et al., Phys. Rev. E 50, 4113 (1994).

16. S. Luding et al., in Fractal Aspects of Materials (Materials Research Society, Symposium Proceedings, Pittsburgh, Pennsylvania, 1995), Vol. 367, pp. 495-500.

17. S. Luding et al., Phys. Rev. E 50, R1762 (1994).

18. F. Radjai and D. E. Wolf, Granular Matter 1, 3 (1998).

19. S. Luding et al., Phys. Rev. E 49, 1634 (1994).

20. P. Deltour and J.-L. Barrat, J. Phys. I France 7, 137 (1997).

21. G. Giese and A. Zippelius, Phys. Rev. E 54, 4828 (1996).

22. T. Aspelmeier, G. Giese, and A. Zippelius, Phys. Rev. E 57, 857 (1998).

23. N. V. Brilliantov, F. Spahn, J. M. Hertzsch, and T. Pöschel, Phys. Rev. E 53, 5382 (1996).

24. O. R. Walton, in Mobile Particulate Systems, edited by E. Guazzelli and L. Oger (Kluwer Academic Publishers, Dordrecht, 1995), p. 367.

25. C. Thornton, Journal of Applied Mechanics 64, 383 (1997).

26. E. Falcon, C. Laroche, S. Fauve, and C. Coste, Eur. Phys. J. B 5, 111 (1998).

27. S. Luding, in Powders 86 Grains 97 (Balkema, Amsterdam, 1997), pp. 373-376.

28. S. Luding, T.A.S.K. Quarterly, Scientific Bulletin of Academic Computer Centre of the Technical University of Gdansk (July, 1998).

29. Theory of simple liquids, edited by J. P. Hansen and I. R. McDonald (Academic Press Limited, London, 1986).
30. E. Falcon, C. Laroche, S. Fauve, and C. Coste, Eur. Phys. J. B 3, 45 (1998).

31. S. Luding, in Physics of dry granular media - NATO ASI Series E350, edited by H. J. Herrmann, J.-P. Hovi, and S. Luding (Kluwer Academic Publishers, Dordrecht, 1998), p. 285.

32. B. D. Lubachevsky, J. of Comp. Phys. 94, 255 (1991).

33. J. D. Goddard, in Recent Developments in Structered Continua. Pitman Research Notes in Mathematics No. 143, edited by D. DeKee and P. N. Kaloni (Longman, J. Wiley, New York, 1986), p. 179.

34. O. R. Walton and R. L. Braun, Journal of Rheology 30 , 949 (1986).

35. J. M. Ziman, Models of Disorder (Cambridge University Press, Cambridge, 1979).

36. S. Luding, M. Huthmann, S. McNamara, and A. Zippelius, Phys. Rev. E 58, 3416 (1998).

37. F. Reif, Fundamentals of Statistical and Thermal Physics (McGraw-Hill, Singapore, 1988).

38. S. Luding and S. McNamara, in preparation (unpublished).

39. I. Goldhirsch and G. Zanetti, Phys. Rev. Lett. 70, 1619 (1993).

40. S. Luding, M. Müller, and S. McNamara, in World Congress on Particle Technology (Institution of Chemical Engineers, Davis Building, 165-189 Railway Terrace, Rugby CV21 3HQ, UK, 1998), ISBN 0-85295-401-9.

41. J. J. Brey, M. J. Ruiz-Montero, and D. Cubero, Phys. Rev. E 54, 3664 (1996)

42. Y. Du, H. Li, and L. P. Kadanoff, Phys. Rev. Lett. 74, 1268 (1995)

43. E. L. Grossman, T. Zhou, and E. Ben-Naim, Phys. Rev. E 55, 4200 (1997).

44. S. McNamara and W. R. Young, Phys. Fluids A 5, 34 (1993).

45. E. L. Grossman and B. Roman, Phys. Fluids 8, 3218 (1996).

46. A. Kudrolli and J. P. Gollub, in Powders \& Grains 97 (Balkema, Rotterdam, 1997), p. 535.

47. A. Kudrolli, M. Wolpert, and J. Gollub, Phys. Rev. Lett. 78, 1383 (1997).

48. M. Sibuya, T. Kawai, and K. Shida, Physica A 167, 676 (1990).

49. E. Trizac and J. P. Hansen, Phys. Rev. Lett. 74, 4114 (1995).

50. F. Spahn, U. Schwarz, and J. Kurths, Phys. Rev. Lett. 78, 1596 (1997)

51. J. A. G. Orza, R. Brito, T. P. C. van Noije, and M. H. Ernst, Int. J. of Mod. Phys. C 8, 953 (1997).

52. E. Trizac and J. P. Hansen, J. Stat. Phys. 82, 1345 (1996).

53. R. Brito and M. H. Ernst, cond-mat/9807224 (unpublished). 